\title{
Geometry-Texture Decomposition/Reconstruction Using a Proximal Interior Point Algorithm
}

\author{
Marie-Caroline Corbineau ${ }^{1}$, Emilie Chouzenoux ${ }^{1,2}$, Jean-Christophe Pesquet $^{1}$ \\ ${ }^{1}$ CVN, CentraleSupélec, INRIA, Université Paris-Saclay, 91190, Gif-Sur-Yvette, France \\ ${ }^{2}$ Université Paris-Est Marne la Vallée, LIGM, UMR CNRS 8049, Champs-sur-Marne, France \\ firstname.lastname@centralesupelec.fr
}

\begin{abstract}
The geometry-texture decomposition of images produced by X-Ray Computed Tomography (CT) is a challenging inverse problem which is usually performed in two steps: reconstruction and decomposition. Decomposition can be used for instance to produce an approximate segmentation of the image, but this one can be compromised by artifacts and noise arising from the acquisition and reconstruction processes. We propose a geometry-texture decomposition based on a TV-Laplacian model, well-suited for segmentation and edge detection. The corresponding joint reconstruction and decomposition task from CT data is then formulated as a convex constrained minimization problem. We use our recently introduced proximal interior point method to solve this inverse problem in a reliable manner. Numerical experiments on realistic images of material samples illustrate the practical efficiency of the proposed approach. Our algorithm indeed compares favorably with a state-of-the-art method.

Index Terms-Geometry-texture decomposition, interior point method, proximal algorithm, computed tomography
\end{abstract}

\section{INTRODUCTION}

Decomposing a natural image $x^{t+g}$ into two components, namely a texture $x^{t}$ and a geometry $x^{g}$ such that $x^{t+g}=$ $x^{t}+x^{g}$, provides solutions to various tasks in image processing such as denoising [1], clustering and classification [2] [3], texture segmentation [4], or digital inpainting [5]. Traditionally, the geometry represents a piecewise smooth version of the image, enabling large object detection, in contrast with the textural component which depicts details and local components with higher spatial frequencies. For instance, in the context of noise removal, the texture represents the noise contribution and consequently shares the same statistical properties, while the geometry can be extracted using the total variation semi-norm [1] [6]. The textural component can also capture meaningful features of an image, corresponding for example to periodic elements, edges, or blobs. A large number of models can be found in the literature regarding the texture extraction using variational approaches, based on $\ell_{1}$ or $\ell_{2}$ regularizations, wavelet decomposition or the Hölder exponent to name only a few [7]-[10]. In this paper, we focus on the use of the Laplacian detector, which has been known for a long time to be useful for texture element extraction, more precisely for edge and blob detection [11]-[13].

In the specific case of material image analysis, the geometrytexture decomposition aims at classifying material components. However, this task can be compromised by the very nature of the samples which can exhibit erosion or microporosity, blurring the border between the different elements. Another issue is the X-Ray Computed Tomography (CT) acquisition procedure used for this type of images. The latter provides a fast non-destructive scanning technique [14]. It consists in measuring the absorption of an object along a sampled grid of size $L$ for $N_{\theta}$ angular positions. This acquisition process can be modelled by a sparse projection operator $H \in \mathbb{R}^{m \times n}$, where $n$ is the number of pixels of the absorption image and $m=L \times N_{\theta}$. Reconstructing the image from the measured data $y=H x^{t+g}$ (or a noisy version of it), called sinogram, is a challenging inverse problem [15] which involves ringing artifacts, contrast issues due to beam hardening, and noise caused by sensor motion [16]. These artifacts can be partially removed by improving scanning techniques or by increasing the number of measurements but this comes at a price and lots of applications can benefit from a faster subsampled CT acquisition. Reconstructing the image from the acquired data is a heavy processing step which can introduce a bias in the subsequent segmentation task [17]. Hence, there is a need for a reliable technique that can allow performing both reconstruction and segmentation on $\mathrm{CT}$ data in a reasonable time.

Joint geometry-texture decomposition and tomographic reconstruction can be formulated as a large-scale convex optimization problem under constraints. Interior point approaches have proven to be very useful and efficient to solve constrained optimization problems [18] [19]. The main idea behind these methods is to replace the constrained problem by an unconstrained minimization problem where the objective function is augmented with a so-called barrier term which goes to infinity as the variable approaches the border of the feasible domain. One advantage of interior point algorithms is that they guarantee feasibility of every iterate, which can boost convergence. However, most of interior point approaches rely on second-order methods [20]-[22] and thus require the objective function to be differentiable. In particular this framework excludes the use of total variation, which is at the core of segmentation variational models.

In this article, we introduce a TV-Laplacian geometry-texture model and propose a compact formulation to perform joint reconstruction and decomposition from tomographic data under some bounded measurement error. The corresponding constrained convex optimization problem is solved using a 
recently introduced proximal interior point method that can handle non-differentiable terms in the objective function. The proposed optimization method leads to conclusive results for images of materials. This article is organized as follows: Section II details the geometry-texture decomposition model and the corresponding inverse problem; the optimization method is described in Section III; Section IV presents numerical experiments carried out on real data and comparison to a stateof-the-art algorithm; a short conclusion is drawn in Section V.

\section{PROBLEM FORMULATION}

\section{A. Notation and definitions}

Let $I_{n} \in \mathbb{R}^{n \times n}, 0_{n} \in \mathbb{R}^{n}$, and $1_{n} \in \mathbb{R}^{n}$ be the identity matrix, the zero vector, and the one vector, respectively. We denote by $\mathcal{S}_{++}^{n}$ the set of positive definite matrices in $\mathbb{R}^{n \times n}$. Let $\Gamma_{0}\left(\mathbb{R}^{n}\right)$ denote the set of functions which are proper, convex, lower semi-continuous on $\mathbb{R}^{n}$ and take values in $\mathbb{R} \cup\{+\infty\}$. The proximity operator [23] is defined as follows: for every $A \in \mathcal{S}_{++}^{n}, f \in \Gamma_{0}\left(\mathbb{R}^{n}\right)$, and $x \in \mathbb{R}^{n}$,

$$
\operatorname{prox}_{f}^{A}(x)=\underset{y \in \mathbb{R}^{n}}{\arg \min }\left(\frac{1}{2}\|y-x\|_{A}^{2}+f(y)\right) .
$$

\section{B. Problem formulation}

We propose to decompose an image into two parts: a geometry and a texture. The geometry is represented by a piecewise constant image which can be viewed as an approximation to the segmented map. Conversely, the texture represents the fastspatially varying components, namely the edges and textural components of the natural image, the residual noise, and artifacts from the tomography measurement. Assuming that the input data $y \in \mathbb{R}^{m}$ is a CT sinogram with a measure uncertainty bounded by $\chi>0$, the following formulation is proposed for the geometry-texture decomposition:

$$
\begin{aligned}
& \mathcal{P}_{0}: \underset{\left(x^{t}, x^{g}\right) \in \mathbb{R}^{n} \times \mathbb{R}^{n}}{\operatorname{minimize}} \frac{1}{2}\left\|F x^{t}\right\|_{2}^{2}+\lambda \operatorname{TV}\left(x^{g}\right) \\
& \text { subject to } \quad x^{t}+x^{g} \in[0,1]^{n} \\
& \left\|H\left(x^{t}+x^{g}\right)-y\right\|_{\infty} \leq \chi
\end{aligned}
$$

where $x^{t}$ is the texture and $x^{g}$ is the geometry, $\lambda>0$ is the regularization parameter, $\mathrm{TV}$ is the isotropic total variation semi-norm [1], $H \in \mathbb{R}^{m \times n}$ is the discrete Radon projection operator, $F=I_{n}-\Delta \in \mathbb{R}^{n \times n}, \Delta \in \mathbb{R}^{n \times n}$ is the Laplacian associated with the $2 \mathrm{D}$ kernel:

$$
\left(\begin{array}{ccc}
0 & 1 & 0 \\
1 & -4 & 1 \\
0 & 1 & 0
\end{array}\right)
$$

padded with circulant assumption. The first term in the criterion enforces edge detection in the texture while the geometry is made smooth thanks to the total variation regularization. The first set of constraints corresponds to the bounds on the pixel values of the natural image $x^{t+g}=x^{t}+x^{g}$, while the second constraint is the data-fitting term which can be decomposed into $2 \times m$ linear inequalities. We introduce the notation $x=\left[\left(x^{t}\right)^{\top}\left(x^{g}\right)^{\top}\right]^{\top}$, and since the constraints are linear, we can define $C \in \mathbb{R}^{2(n+m) \times 2 n}$ and $c \in \mathbb{R}^{2(n+m)}$ such that $x$ satisfies the constraints if and only if $C x+c \in]-\infty, 0]^{2(n+m)}$ where

$$
C=\left(\begin{array}{cc}
-I_{n} & -I_{n} \\
I_{n} & I_{n} \\
-H & -H \\
H & H
\end{array}\right) \quad \text { and } \quad c=\left(\begin{array}{c}
0_{n} \\
-1_{n} \\
-\chi 1_{m}+y \\
-\chi 1_{m}-y
\end{array}\right)
$$

\section{OPTIMIZATION METHOD}

We propose to solve the constrained problem $\mathcal{P}_{0}$ via a sequence $\left(\mathcal{P}_{\mu_{j}}\right)_{j \in \mathbb{N}}$ of unconstrained subproblems parametrized by the barrier coefficient $\mu_{j}$ and defined as follows:

$$
\mathcal{P}_{\mu_{j}}: \underset{\left(x^{t}, x^{g}\right) \in \mathbb{R}^{n} \times \mathbb{R}^{n}}{\operatorname{minimize}} \frac{1}{2}\left\|F x^{t}\right\|_{2}^{2}+\lambda \operatorname{TV}\left(x^{g}\right)+\mu_{j} b(C x+c),
$$

where $b$ is the logarithmic barrier: $\left(\forall z=\left(z_{i}\right)_{1 \leq i \leq 2(n+m)} \in\right.$ $\left.\mathbb{R}^{2(n+m)}\right) b(z)=-\sum_{i=1}^{2(m+n)} \ln \left(-z_{i}\right)$ if $\left.z \in\right]-\infty, 0\left[\left[^{2(n+m)}\right.\right.$, $+\infty$ otherwise. As the subproblems $\left(\mathcal{P}_{\mu_{j}}\right)_{j \in \mathbb{N}}$ do not have an explicit solution, we solve them approximately using proximal gradient iterations, leading to the generic Proximal Interior Point Algorithm (PIPA) introduced in [24], the specific implementation of which is detailed below.

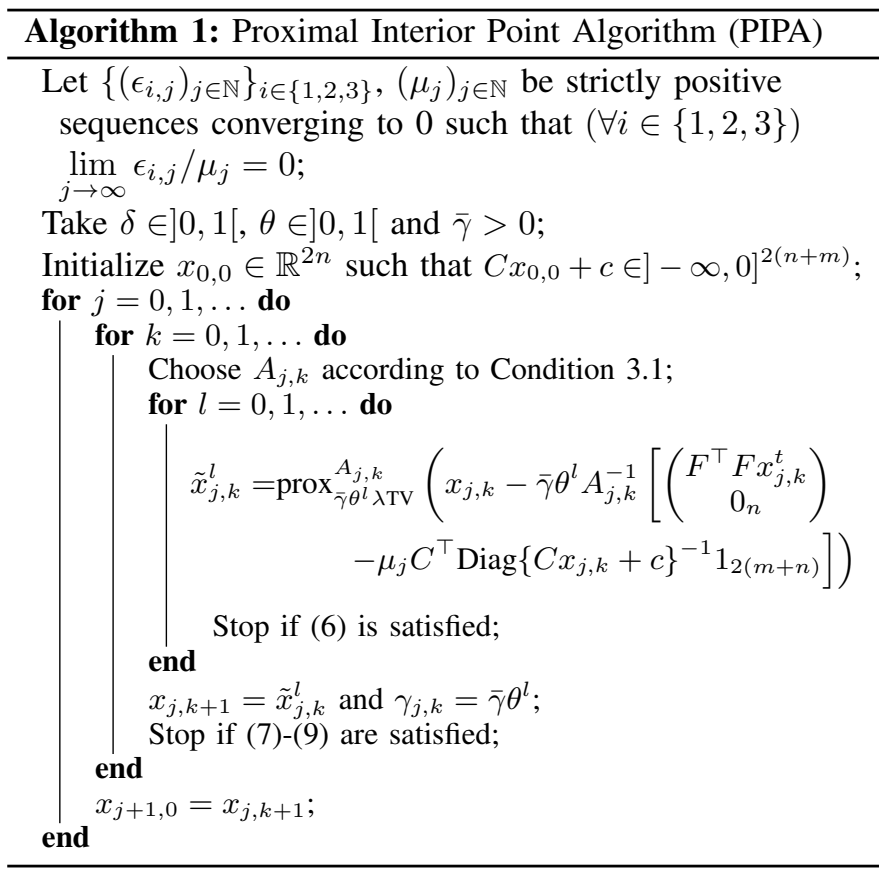

Given a fixed barrier parameter $\mu_{j}>0, \mathcal{P}_{\mu_{j}}$ is solved approximately via several forward-backward iterations [25] [26] which include a gradient step on the barrier and on the texture regularization, and a proximal step on the total variation term. In order to accelerate the convergence rate, the proximity operator is associated to a preconditioning matrix $A_{j, k}$ which should satisfy Condition 3.1 below. The choice for this matrix is detailed in Section III-B.

Condition 3.1: For every $j \in \mathbb{N}\left(\exists\left(\underline{\nu}_{j}, \bar{\nu}_{j}\right) \in\right] 0,+\infty\left[^{2}\right)$ such that $(\forall k \in \mathbb{N}) A_{j, k} \in \mathcal{S}_{++}^{n}$ and $\underline{\nu}_{j} I_{n} \preceq A_{j, k} \preceq \bar{\nu}_{j} I_{n}$. 
Since the barrier is not Lipschitz differentiable, a linesearch is performed to find an appropriate stepsize $\gamma_{j, k}$ [27] [28]. The backtracking is based on the stopping criterion given by

$$
\begin{aligned}
& \frac{1}{2}\left\|F\left(\tilde{x}_{j, k}^{l}-x_{j, k}\right)^{t}\right\|_{2}^{2}+\mu_{j}\left(b\left(C \tilde{x}_{j, k}^{l}+c\right)-b\left(C x_{j, k}+c\right)\right) \\
& +\mu_{j} \sum_{i=1}^{2(m+n)} \frac{\left(C\left(\tilde{x}_{j, k}^{l}-x_{j, k}\right)\right)_{i}}{\left(C x_{j, k}+c\right)_{i}} \leq \frac{\delta}{\bar{\gamma} \theta^{l}}\left\|\tilde{x}_{j, k}^{l}-x_{j, k}\right\|_{A_{j, k}}^{2} .
\end{aligned}
$$

The barrier parameter $\mu_{j}$ is decreased as soon as the following accuracy conditions are met:

$$
\begin{gathered}
\left\|x_{j, k}-x_{j, k+1}\right\| \leq \epsilon_{1, j} \\
\frac{1}{\gamma_{j, k}}\left\|A_{j, k}\left(x_{j, k}-x_{j, k+1}\right)\right\| \leq \epsilon_{2, j} \\
\sum_{i=1}^{2(n+m)}\left|\frac{\left(C x_{j, k+1}+c\right)_{i}}{\left(C x_{j, k}+c\right)_{i}}-1\right| \leq \epsilon_{3, j} .
\end{gathered}
$$

As stated in [24], the sequence $\left(x_{j, 0}\right)_{j \in \mathbb{N}}$ produced by PIPA is bounded and every cluster point of it is a solution to $\mathcal{P}_{0}$.

\section{A. Initialization}

The proposed interior point method must be initialized in the strict interior of the feasible domain. To this aim, we initialize $x^{t}$ with the zero vector and follow the method of [29, Chap. 11.4] for $x^{g}$. We consider the minimization of the maximal infeasibility:

$$
\begin{aligned}
& \mathcal{P}_{I}: \underset{\left(s, x^{g}\right) \in \mathbb{R} \times \mathbb{R}^{n}}{\operatorname{minimize}} s \\
& \text { subject to } \quad s \geq 0, x^{g} \in[0,1]^{n} \\
& \left\|H x^{g}-y\right\|_{\infty} \leq \chi+s
\end{aligned}
$$

which is solved using a standard primal-dual interior point algorithm. This iterative process is stopped once the constraints in $\mathcal{P}_{0}$ are strictly satisfied or when $s$ is sufficiently small. In all the numerical experiments we performed, we were able to initialize our method using this procedure in a reasonable time.

\section{B. Diagonal preconditioning matrix}

For the traditional forward-backward algorithm, a suitable preconditioning matrix $A_{j, k}$ can be chosen as an approximation of the Hessian of the differentiable term in (5) at the current iterate [30], which, in our case, is of the form

$$
\left(\begin{array}{cc}
F^{\top} F+G\left(x_{j, k}\right) & G\left(x_{j, k}\right) \\
G\left(x_{j, k}\right) & G\left(x_{j, k}\right)
\end{array}\right)
$$

where

$$
\begin{aligned}
G\left(x_{j, k}\right)= & \mu_{j} D_{1}\left(x_{j, k}\right)+\mu_{j} H^{\top} D_{2}\left(x_{j, k}\right) H \\
D_{1}\left(x_{j, k}\right)= & \operatorname{Diag}\left\{x_{j, k}^{t+g}\right\}^{-2}+\operatorname{Diag}\left\{1_{n}-x_{j, k}^{t+g}\right\}^{-2} \\
D_{2}\left(x_{j, k}\right)= & \operatorname{Diag}\left\{H x_{j, k}^{t+g}+\chi 1_{m}-y\right\}^{-2} \\
& \quad+\operatorname{Diag}\left\{-H x_{j, k}^{t+g}+\chi 1_{m}+y\right\}^{-2} .
\end{aligned}
$$

Given the huge size and ill-conditioning of $H$, the inverse of $H^{\top} D_{2}\left(x_{j, k}\right) H$ is hardly computable. Hence, instead of using the full Hessian we propose to rely on the diagonal approximation proposed in [31] for $G\left(x_{j, k}\right)$, so that:

$$
A_{j, k}=\left(\begin{array}{cc}
F^{\top} F+D\left(x_{j, k}\right) & D\left(x_{j, k}\right) \\
D\left(x_{j, k}\right) & D\left(x_{j, k}\right)
\end{array}\right)
$$

where

$$
D\left(x_{j, k}\right)=\mu_{j} D_{1}\left(x_{j, k}\right)+\mu_{j} \operatorname{Diag}\left\{P^{\top} d_{2}\left(x_{j, k}\right)\right\}
$$

and $d_{2}\left(x_{j, k}\right) \in \mathbb{R}^{m}$ is the diagonal of $D_{2}\left(x_{j, k}\right), P \in \mathbb{R}^{m \times n}$ with $(\forall(i, \ell))[P]_{i, \ell}=[H]_{i, \ell} \sum_{q=1}^{n}[H]_{i, q}$. The operator $A_{j, k}$ is easily invertible since $D\left(x_{j, k}\right)$ is diagonal.

\section{NUMERICAL RESUlTS AND DISCUSSION}

The efficiency of the proposed approach is illustrated through the reconstruction and decomposition of three natural images from material samples, depicted in Figure 1. These images of size $n=128 \times 128$ present various textural elements and artifacts. The discrete Radon operator $H \in \mathbb{R}^{m \times n}$ corresponds to projections along $N_{\theta}=180$ angular positions on a grid of size $L=128$, so that $m=180 \times 128$. To mimick measurement uncertainty, the sinograms are corrupted with a uniform noise of amplitude $\chi=1$. The regularization parameter $\lambda$ is chosen for each image, such that it leads to satisfactory geometry estimates: it is fixed to 0.6 for the glass sample, 0.1 for the $\mathrm{AlCu}$ data, and to 0.1 for the basalt image. Note that the correlation criterion defined in [7] could also have been used to assess the decomposition. The proposed algorithm PIPA is compared to the standard Alternating Direction Method of Multipliers (ADMM) [32]. Since the latter does not need to be initialized in the feasible domain, we run it with two different initializations, namely the one that we use for PIPA (labeled ADMM1) and the zero vector (labeled ADMM2).

The methods are implemented on Matlab R2016b and the simulations are performed on a desktop computer with an Intel Xeon $3.2 \mathrm{GHz}$ processor and $16 \mathrm{~GB}$ of RAM.

\section{A. Results}

The performance of the different optimization algorithms is evaluated in terms of the normalized distance from the current iterate $x_{j, k}$ to the final point $x_{\infty}$, where $x_{\infty}$ is obtained after running the algorithms for $20000 \mathrm{~s}$. The corresponding plots
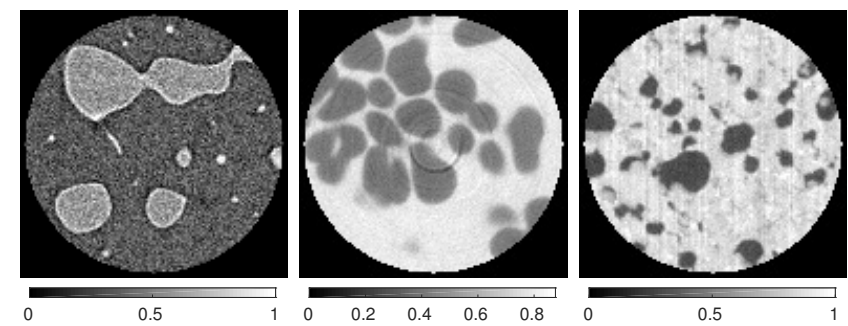

Figure 1. Natural images. (left and middle) Phase-separated barium borosilicate glass and $\mathrm{AlCu}$ sample, resp., imaged at the ESRF synchrotron (courtesy of David Bouttes). (right) Colorado Plateau basalt with bubbles (courtesy of Alexander Proussevitch [33]) 

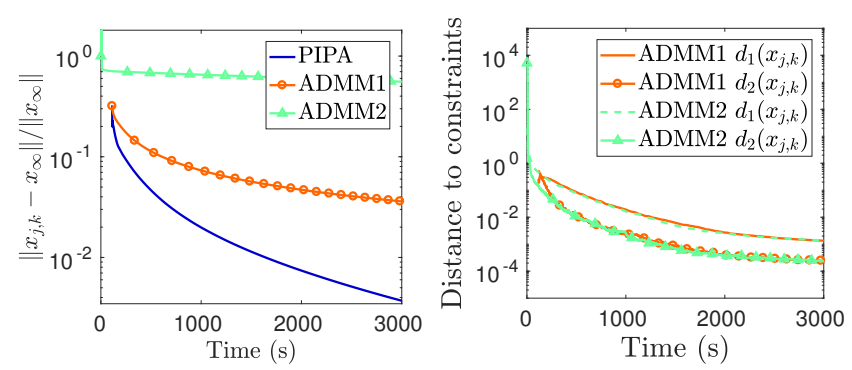

Figure 2. Glass sample: (left) distance from the current iterate to the final point, (right) distance to the constraints with time.
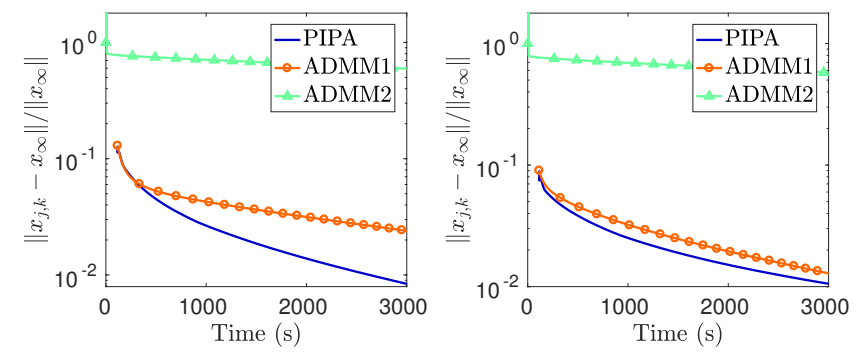

Figure 3. Distance from the current iterate to the final point: (left) $\mathrm{AlCu}$ data, (right) basalt sample.

can be found on Figure 2 (left) and Figure 3. Note that the time needed to solve $\mathcal{P}_{I}$ for initializing PIPA and ADMM1 is included into these graphs, which corresponds to a duration of about 115 seconds for all three tests.

Moreover, Figure 2 (right) represents the distance from the iterates to the constraints for ADMM1 and ADMM2 where $d_{1}\left(x_{j, k}\right)$ and $d_{2}\left(x_{j, k}\right)$ are the distances from $x_{j, k}^{t+g}$ to $[0,1]^{n}$ and from $H x_{j, k}^{t+g}-y$ to $[-\chi, \chi]^{m}$, respectively.

The proposed method efficiency is also assessed according to the visual results given by the decomposition. We consider that PIPA has almost converged when $\left\|x_{j, k}-x_{\infty}\right\| /\left\|x_{\infty}\right\|$ is below 0.01 . This accuracy is reached after $20 \mathrm{~min}, 39 \mathrm{~min}$ and 44 min for the glass, $\mathrm{AlCu}$ and basalt data, respectively. The corresponding texture, geometry and reconstructed images can be found on Figure 4. The quality of the reconstructed images can be evaluated according to the signal to noise ratio (SNR) defined by $\mathrm{SNR}=-20 \log _{10}\left(\left\|x_{j, k}^{t+g}-\bar{x}\right\| /\|\bar{x}\|\right)$ where $\bar{x}$ is the groundtruth (see Figure 1). The SNR for Figure 4 (last column) is equal to $14.7 \mathrm{~dB}, 27.8 \mathrm{~dB}$ and $25.7 \mathrm{~dB}$ (from top to bottom).

\section{B. Discussion}

In terms of optimization speed, as one can see on Figures 2 (left) and 3, the proposed algorithm converges faster to its limit point. In addition, both PIPA and ADDM1 perform better than ADMM2, which shows that initializing the algorithms with the solution obtained by solving $\mathcal{P}_{I}$ is significantly beneficial. It should also be noted that PIPA and ADMM do not follow the same path: as opposed to the proposed interior point algorithm, ADMM does not produce feasible iterates (even if they converge to one). This is confirmed by Figure 2 (right). Regarding Figure 4, the proposed decomposi-
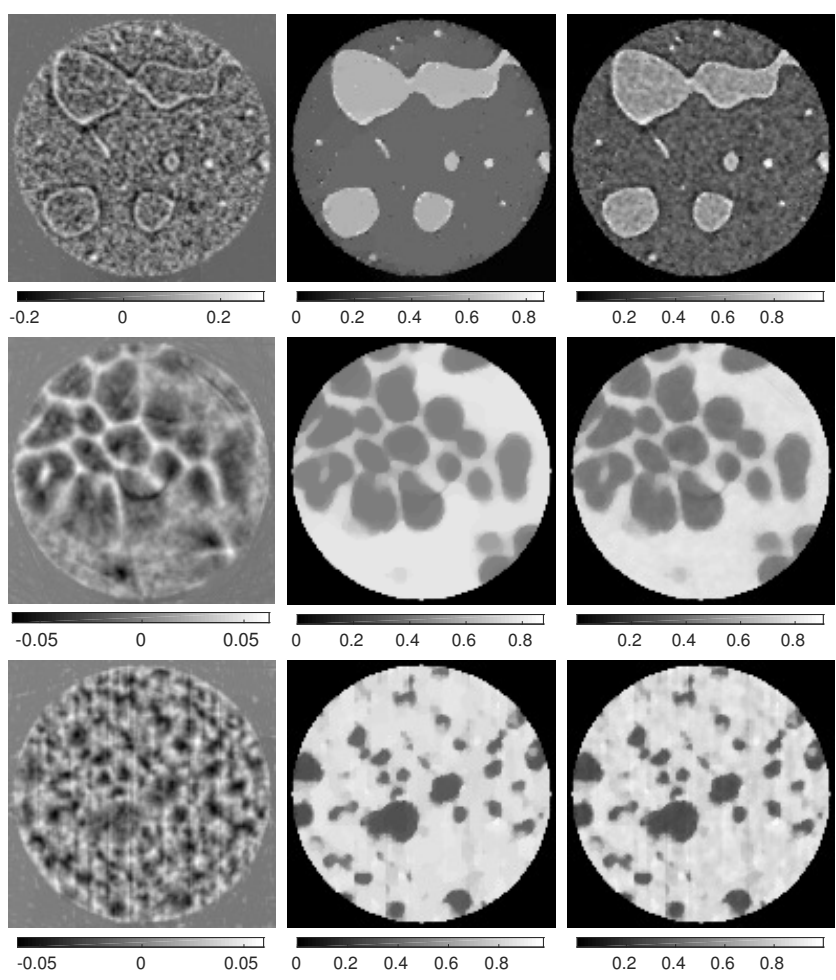

Figure 4. Visual results obtained with PIPA. (rows from top to bottom) Glass sample, $\mathrm{AlCu}$, basalt. (columns from left to right) Texture, geometry, reconstruction: sum of texture and geometry.

tion scheme provides visually satisfactory results. For the glass sample, the geometry, which is free of the noisy background and of the textural elements on the borders between the two materials, approximates well the expected segmentation, while the texture captures the edges between the different areas of the sample. The $\mathrm{AlCu}$ data is characterized by ringing artifacts which are almost removed from the geometry. One may expect that the analysis of this sample content would be improved by combining information from the geometry and the texture which highlights significantly the edges between the different blobs. The basalt image is affected by vertical artifacts along with blur. As expected, these vertical lines can be found in the resulting texture, leaving the geometry smooth and relevant for a two-phase classification step.

\section{CONCLUSION}

In this work, we proposed a TV-Laplacian geometry-texture model for image reconstruction from tomographic measurements. We formulated the joint decomposition and tomographic reconstruction task as a constrained optimization problem. A novel proximal interior point algorithm was proposed to solve this problem, that compares favorably with ADMM in terms of convergence speed. In addition, it allows meaningful decomposition results to be obtained when tested on realistic test images of materials. One possible improvement of our approach could be to consider more sophisticated non-linear constraints and to employ a data-fitting term better adapted to the noise statistics encountered in given acquisition processes. 


\section{REFERENCES}

[1] L. I. Rudin, S. Osher, and E. Fatemi, "Nonlinear total variation based noise removal algorithms," Physica D: Nonlinear Phenomena, vol. 60, no. 1-4, pp. 259-268, 1992.

[2] G. Evangelopoulos and P. Maragos, "Image decomposition into structure and texture subcomponents with multifrequency modulation constraints," in Proc. of the Computer Society Conference on Computer Vision and Pattern Recognition (CVPR 2008). Achorage, USA: IEEE, 23-28 June 2008, pp. 1-8.

[3] J.-F. Aujol and T. F. Chan, "Combining geometrical and textured information to perform image classification," Journal of Visual Communication and Image Representation, vol. 17, no. 5, pp. 1004-1023, 2006.

[4] J. Frecon, N. Pustelnik, H. Wendt, L. Condat, and P. Abry, "Multifractalbased texture segmentation using variational procedure," in Proc. of the 12th Image, Video, and Multidimensional Signal Processing Workshop (IVMSP 2016). IEEE, 11-12 July 2016, pp. 1-5.

[5] M. Bertalmio, L. Vese, G. Sapiro, and S. Osher, "Simultaneous structure and texture image inpainting," IEEE Transactions on Image Processing, vol. 12 , no. 8, pp. 882-889, 2003.

[6] S. Osher, A. Solé, and L. Vese, "Image decomposition and restoration using total variation minimization and the $\mathrm{H}^{-1}$ norm," Multiscale Modeling \& Simulation, vol. 1, no. 3, pp. 349-370, 2003.

[7] J.-F. Aujol, G. Gilboa, T. Chan, and S. Osher, "Structure-texture image decomposition-modeling, algorithms, and parameter selection," International Journal of Computer Vision, vol. 67, no. 1, pp. 111-136, 2006.

[8] L. M. Briceño-Arias, P. L. Combettes, J.-C. Pesquet, and N. Pustelnik, "Proximal method for geometry and texture image decomposition," in Proc. of the 17th International Conference on Image Processing (ICIP 2010). Hong Kong, HK: IEEE, 26-29 September 2010, pp. 2721-2724.

[9] L. M. Briceño-Arias, P. L. Combettes, J.-C. Pesquet, and N. Pustelnik, "Proximal algorithms for multicomponent image recovery problems," Journal of Mathematical Imaging and Vision, vol. 41, no. 1, pp. 3-22, 2011.

[10] N. Pustelnik, H. Wendt, and P. Abry, "Local regularity for texture segmentation: Combining wavelet leaders and proximal minimization," in Proc. of the 38th International Conference on Acoustics, Speech, and Signal Processing (ICASSP 2013). Vancouver, CAN: IEEE, 26-31 May 2013, pp. pp-5348.

[11] R. M. Haralick, "Statistical and structural approaches to texture," Proc. of the IEEE, vol. 67, no. 5, pp. 786-804, 1979.

[12] D. Blostein and N. Ahuja, "A multiscale region detector," Computer Vision, Graphics, and Image Processing, vol. 45, no. 1, pp. 22-41, 1989.

[13] T. Lindeberg, "Feature detection with automatic scale selection," International Journal of Computer Vision, vol. 30, no. 2, pp. 79-116, 1998.

[14] C. A. Kak and M. Slaney, Principles of computerized tomographic imaging. IEEE Press, 1988.

[15] E. Chouzenoux, F. Zolyniak, E. Gouillart, and H. Talbot, "A majorizeminimize memory gradient algorithm applied to X-ray tomography." in Proc. of the 20th International Conference on Image Processing (ICIP 2013). Melbourne, AUS: IEEE, 15-18 September 2013, pp. 1011-1015.

[16] R. A. Ketcham and W. D. Carlson, "Acquisition, optimization and interpretation of X-ray computed tomographic imagery: applications to the geosciences," Computers \& Geosciences, vol. 27, no. 4, pp. 381-400, 2001.

[17] E. Gouillart, F. Krzakala, M. Mezard, and L. Zdeborová, "Beliefpropagation reconstruction for discrete tomography," Inverse Problems, vol. 29, no. 3, p. 035003, 2013.

[18] M. H. Wright, "Interior methods for constrained optimization," Acta Numerica, vol. 1, pp. 341-407, 1991.

[19] J. Gondzio, "Interior point methods 25 years later," European Journal of Operational Research, vol. 218, no. 3, pp. 587-601, 2012.

[20] A. Forsgren, P. E. Gill, and M. H. Wright, "Interior methods for nonlinear optimization," SIAM Review, vol. 44, no. 4, pp. 525-597, 2002.

[21] P. Armand, J. C. Gilbert, and S. Jan-Jégou, "A feasible BFGS interior point algorithm for solving convex minimization problems," SIAM Journal on Optimization, vol. 11, no. 1, pp. 199-222, 2000.

[22] S. Bonettini and T. Serafini, "Non-negatively constrained image deblurring with an inexact interior point method," Journal of Computational and Applied Mathematics, vol. 231, no. 1, pp. 236-248, 2009.

[23] H. H. Bauschke and P. L. Combettes, Convex analysis and monotone operator theory in Hilbert spaces. Springer New York, 2011.
[24] M.-C. Corbineau, E. Chouzenoux, and J.-C. Pesquet, "PIPA: a new proximal interior point algorithm for large-scale convex optimization," in Proc. of the 43rd International Conference on Acoustics, Speech and Signal Processing (ICASSP 2018). Calgary, CAN: IEEE, 15-20 April 2018 (forthcoming).

[25] P. L. Combettes and V. R. Wajs, "Signal recovery by proximal forwardbackward splitting," Multiscale Modeling \& Simulation, vol. 4, no. 4, pp. 1168-1200, 2005

[26] P. L. Combettes and J.-C. Pesquet, "Proximal splitting methods in signal processing," in Fixed-point algorithms for inverse problems in science and engineering. Springer, 2011, pp. 185-212.

[27] E. Chouzenoux, S. Moussaoui, and J. Idier, "Majorize-minimize linesearch for inversion methods involving barrier function optimization," Inverse Problems, vol. 28, no. 6, p. 065011, 2012.

[28] S. Salzo, "The variable metric forward-backward splitting algorithm under mild differentiability assumptions," SIAM Journal on Optimization, vol. 27, no. 4, pp. 2153-2181, 2017.

[29] S. Boyd and L. Vandenberghe, Convex optimization. Cambridge University Press, 2004.

[30] S. Becker and J. Fadili, "A quasi-newton proximal splitting method," in Proc. of the 25th Advances in Neural Information Processing Systems Conference (NIPS 2012), Lake Tahoe, USA, 3-8 December 2012, pp. 2618-2626.

[31] E. Chouzenoux, J.-C. Pesquet, and A. Repetti, "Variable metric forwardbackward algorithm for minimizing the sum of a differentiable function and a convex function," Journal of Optimization Theory and Applications, vol. 162, no. 1, pp. 107-132, 2014.

[32] S. Setzer, G. Steidl, and T. Teuber, "Deblurring Poissonian images by split Bregman techniques," Journal of Visual Communication and Image Representation, vol. 21, no. 3, pp. 193-199, 2010.

[33] A. A. Proussevitch, D. L. Sahagian, and W. D. Carlson, "Statistical analysis of bubble and crystal size distributions: application to Colorado Plateau basalts," Journal of Volcanology and Geothermal Research, vol. 164, no. 3, pp. 112-126, 2007. 